BRAVZULIAN JOURNAL

OF MEDICAL AND BIOLOGICAL RESFARCH

www.bjournal.com.br
ISSN 0100-879X

Volume 43 (5) 381-496 May 2011

BIOMEDICAL SCIENCES

AND

CLINICAL INVESTIGATION

Braz J Med Biol Res, May 2011, Volume 44(5) 418-420

doi: 10.1590/S0100-879X2011007500036

Novel regulatory SNPs in the promoter region of the TNFRSF18 gene in a Gabonese population

T.P. Velavan, S. Bechlars, X. Huang, P.G. Kremsner and J.F.J. Kun

The Brazilian Journal of Medical and Biological Research is partially financed by

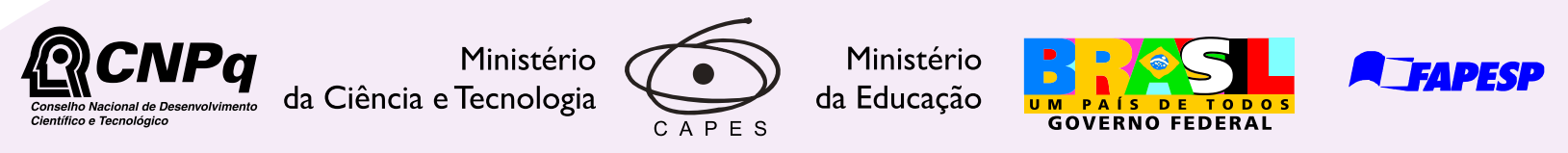

Institutional Sponsors
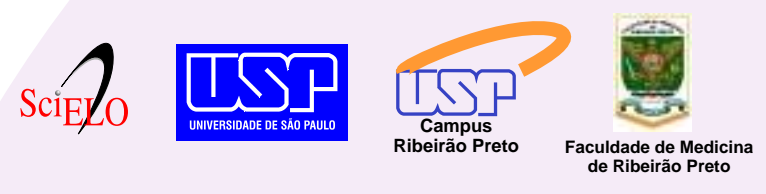

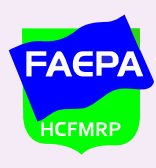

Ф SHIMADZU

GE Healthcare
Hotsite of proteomics metabolomics developped by:

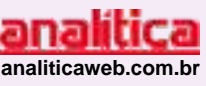

Thermo SCIENTIFIC 


\title{
Novel regulatory SNPs in the promoter region of the TNFRSF18 gene in a Gabonese population
}

\author{
T.P. Velavan ${ }^{1}$, S. Bechlars ${ }^{1}$, X. Huang ${ }^{1}$, P.G. Kremsner ${ }^{1,2}$ and J.F.J. Kun ${ }^{1}$ \\ ${ }^{1}$ Institute for Tropical Medicine, University of Tübingen, Tübingen, Germany \\ ${ }^{2}$ Medical Research Unit, Albert Schweitzer Hospital, Lambaréné, Gabon
}

\begin{abstract}
Parasites are accountable for driving diversity within immune gene families. We identified and investigated regulatory single nucleotide polymorphisms (SNPs) in the promoter regions of the tumor necrosis factor receptor superfamily member 18 (TNFRSF18) gene by direct sequencing in a group of male Gabonese individuals exposed to a wide array of parasitic diseases such as malaria, filariasis and schistosomiasis. Two new promoter variants were identified in 40 individuals. Both novel variants were heterozygous and were linked to SNP \#rs3753344 (C/T), which has been described. One of the SNP variants (ss2080581728) was close to the general transcription factor site, the TATA box. We further validated these new promoter variants for their allelic gene expression using transient transfection assays. One new promoter variant with two base changes (C/T - ss2080581728/ rs3753344) displayed an altered expression of the marker gene. Both novel variants remained less active at the non-induced state in comparison to the major allele. The allele frequencies observed in this study were consistent with data for other African populations. The detection and analysis of these human immune gene polymorphisms contribute to a better understanding of the interaction between host-parasite and expression of Treg activity.
\end{abstract}

Key words: Tumor necrosis factor receptor superfamily; Polymorphism; Transfection; Regulatory T cells

\section{Introduction}

Failure of the immune system to clear parasites may lead to prolonged disease or exacerbated pathology. One reason for this failure may lie in the immunomodulatory effects induced by parasites, which then favor the expansion of regulatory $\mathrm{T}$ cells (Tregs). Tregs are actively involved in the control of pathological and physiological immune responses, thereby contributing to the maintenance of immunological self-tolerance and immune homeostasis (1-3). Regulatory single nucleotide polymorphism (SNPs) can cause significant changes in gene expression in functional immune genes. Promoter regions are potential candidates for the presence of such functional SNPs, as they are involved in transcription initiation and many of the cis-acting elements that regulate gene expression possibly harbor functional polymorphisms (4). Investigation of these human polymorphisms in the promoter region of immune genes possibly reflects the level of susceptibility to parasitic infection and of Treg expression. We investigated the tumor necrosis factor receptor superfamily member 18
(TNFRSF18) locus, a 228-amino acid type I transmembrane protein known to play a key role in the regulation of $\mathrm{T}$ cell receptor-mediated cell death (5). In the current study, the occurrence of different promoter variants inducing differential expression of TNFRSF18 was validated using transient transfection assays.

\section{Material and Methods}

Forty DNA samples obtained from unrelated Gabonese individuals were screened for SNPs in the promoter region of the TNFRSF18 by the polymerase chain reaction (PCR) and subsequent sequencing. The study was approved by the local Ethics Committee of the International Foundation of the Albert Schweitzer Hospital. Fragments of the promoter of the TNFRSF18 genes were amplified using the primer pairs - TNFRSF18-forward: 5'-GCGCCTCTTTCT ATCCAGG-3', TNFRSF18-Intern01: 5'-GGCTCCTCC TCAACTCCCTCC-3', and with TNFRSF18-reverse:

Correspondence: T.P. Velavan, Institute for Tropical Medicine, University of Tübingen, Wilhelmstrasse 27, 72074 Tübingen, Germany. Fax: +49-7071-29-4684. E-mail: velavan@medizin.uni-tuebingen.de

Received July 9, 2010. Accepted March 10, 2011. Available online March 25, 2011. Published May 16, 2011. 
5'AACGCCGGTCTGAGCAC-3' (MWG Operon, Germany). PCR amplifications were carried out in $20-\mu \mathrm{L}$ reaction volumes with $5 \mathrm{ng}$ genomic DNA, 1X PCR buffer $(20 \mathrm{mM}$ Tris- $\mathrm{HCl}, \mathrm{pH} 8.4,50 \mathrm{mM} \mathrm{KCl}$, and $1.5 \mathrm{mM} \mathrm{MgCl}_{2}$; Qiagen, Germany), $0.125 \mathrm{mM}$ dNTPs, $0.5 \mathrm{mM}$ of each primer, and $1 \mathrm{U}$ Taq DNA polymerase (Qiagen) in a PTC-200 Thermal cycler (MJ Research, USA). Thermal cycling parameters were: initial denaturation at $94^{\circ} \mathrm{C}$ for $2 \mathrm{~min}$, followed by 34 cycles of $30 \mathrm{~s}$ at $94^{\circ} \mathrm{C}$ denaturation, $30 \mathrm{~s}$ at $61^{\circ} \mathrm{C}$ annealing temperature, $1 \mathrm{~min}$ and $30 \mathrm{~s}$ at $72^{\circ} \mathrm{C}$ extension, followed by a final extension of $2 \min$ at $72^{\circ} \mathrm{C}$. The PCR products $(1$ $\mu \mathrm{L}$ ) were used directly as templates for sequencing, using the BigDye terminator v. 2.0 cycle sequencing kit (Applied Biosystems, USA) on an ABI 3100 DNA sequencer. Using the program BioEdit (http://www.mbio.ncsu.edu/BioEdit/ bioedit.html), polymorphisms were identified when assembled with the reference sequence of the TNFRSF18 gene obtained from the SNPper database (http://snpper. chip.org/). The respective genomic DNA identified to have SNPs was amplified with infusion primers and were cloned to the pGL3 basic vector using the infusion advantage PCR cloning kit (Clontech, USA). Two independent colonies were picked from these transformations and maxi prep was performed using the Endofree plasmid maxi kit (Qiagen). We tested the activities of the observed polymorphic promoters in unstimulated Jurkat T cell lines. In principle, five independent transfection experiments for each construct were performed in duplicate with Jurkat $T$ cells using the TransIT-Jurkat Transfection Reagent (Mirus Bio Corporation, USA) as recommended by the manufacturer. Data were analyzed by the StatView software (http://www.statview. com). The activity of the TNFRSF18 promoter variants was compared to the activity of the major allele based on their luciferase activities. $P$ values were calculated by ANOVA corrected by the Bonferroni/Dunn test. The differences were considered to be significant when $\mathrm{P} \leq 0.05$.

Table 1. Genetic variants identified in the promoter regions of the TNFRSF18 locus.

\begin{tabular}{lclcccc}
\hline SNP $(\mathrm{rs} \#)$ & Polymorphism & Flanking sequences & Genotype & Analyzed individuals & Allele & Frequency \\
\hline ss2080581724 & C>T & GGAAC[C/T]GCCTT & CC & 39 & C & 0.975 \\
& & & CT & 1 & T & 0.025 \\
ss2080581728 & C>T & ATAAA[C/T]GCCGC & CC & 39 & C & 0.975 \\
& & & CT & 1 & T & 0.025 \\
rs3753344 & C $>$ T & ATCCC[C/T]GCCAG & CC & 34 & C & 0.850 \\
& & & CT & 6 & T & 0.150 \\
\hline
\end{tabular}

SNP = single nucleotide polymorphism .

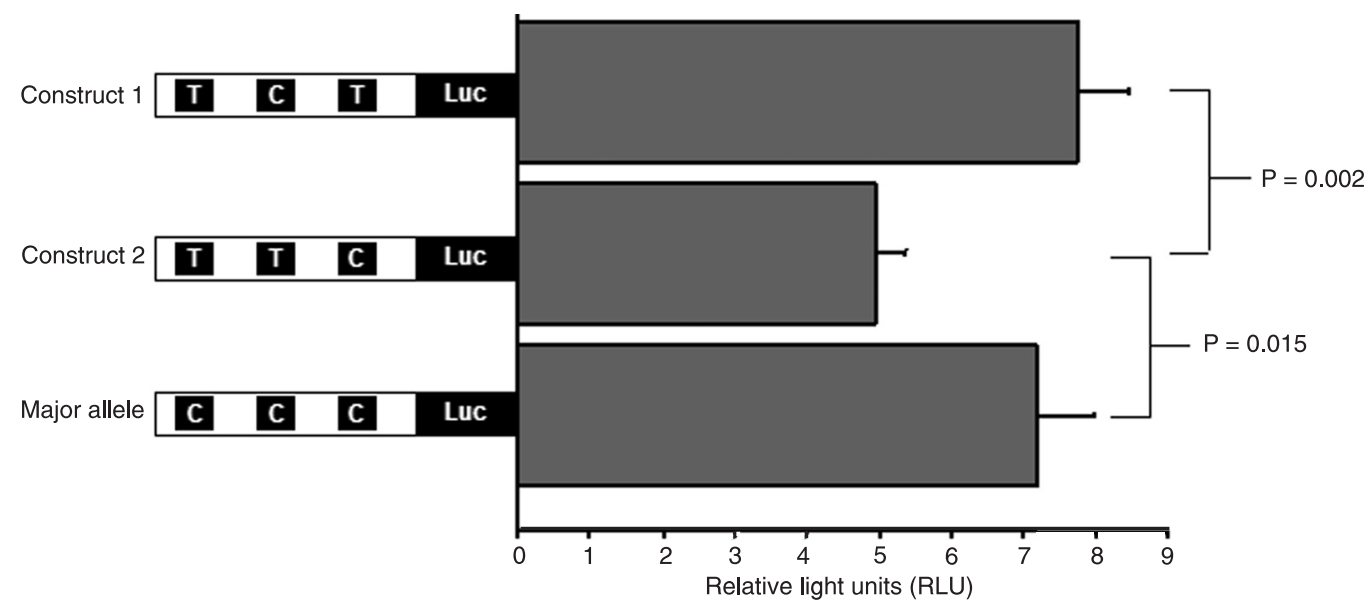

Figure 1. Comparison of the activity of the three different TNFRSF18 promoter variants analyzed based on luciferase (LuC) activity. The ratio of the relative light units (firefly/renilla) is presented. $P$ values were calculated by ANOVA corrected by the Bonferroni/Dunn test. The letters indicate the presence of C/T mutations. 


\section{Results}

Three SNPs were identified in 40 subjects. Two novel SNPs were identified at position \#ss2080581724 (C/T) and \#ss2080581728 (C/T) and an SNP that has been described earlier in the SNPper database was identified at position \#rs3753344 (C/T). Their respective genotype and allelic frequencies are summarized in Table 1. All three SNPs observed were heterozygous. Both the novel SNPs were linked to the described SNP \#rs3753344 (C/T). Comparison of the activity of all three TNFRSF18 promoter variants based on luciferase activity indicated decreased activity for one variant compared to the Major allele (Figure 1). The $\mathrm{P}$ values were significant for Construct 2 (\#ss2080581728/\#rs3753344) compared to the major allele $(P=0.015)$. The $P$ values indicated statistical significance when Construct 1 (\#ss2080581724/\#rs3753344) was compared to construct 2 (\#ss2080581728/\#rs3753344; $P=0.002)$.

\section{Discussion}

In the current study, we identified two novel promoter variants in the TNFRSF18 gene locus. A study in the context of regulatory polymorphisms in the TNF receptor superfamily had reported a similar polymorphism (\#rs3753344 C/T) with an allele frequency of $10 \%$ among 24 Korean individuals (6)

\section{References}

1. Sakaguchi S. Naturally arising CD4+ regulatory $T$ cells for immunologic self-tolerance and negative control of immune responses. Annu Rev Immunol 2004; 22: 531-562.

2. Sakaguchi S. Naturally arising Foxp3-expressing CD25+ CD4+ regulatory $T$ cells in immunological tolerance to self and non-self. Nat Immunol 2005; 6: 345-352.

3. Miyara M, Sakaguchi S. Natural regulatory T cells: mechanisms of suppression. Trends Mol Med 2007; 13: 108-116.

4. Hoogendoorn B, Coleman SL, Guy CA, Smith K, Bowen T, Buckland PR, et al. Functional analysis of human promoter and an allele frequency of $20 \%$ was observed in Japanese individuals as obtained from the JSNP database (http://www. snp.ims.u-tokyo.ac.jp). The current study revealed that the SNPs (\#rs3753344 C/T) contribute to $15 \%$ of allelic distribution among 40 Gabonese individuals, in agreement with the allele frequencies of individuals from an Afro-American ethnic background (http://snpper.chip.org). In the Nigerian Yorubas, both alleles occur at a frequency of 0.5 compared to the $\mathrm{NCBI}$ HapMap database. It appears that a significant difference in the distribution of SNP allele frequencies occurs among diverse ethnic groups, possibly reflecting the role of these regulatory SNPs in confined infectious challenges. In order to associate any disease severity with any of these newly identified SNPs, a very large cohort of samples is obligatory. The regulatory SNPs identified in the current study will provide useful information for understanding the relevance of sequence polymorphisms in populations of different ethnic background and may serve as a basis to study parasite susceptibility in association studies.

\section{Acknowledgments}

We thank A. Weierich, V. Galinat and V. Grummes from Institute for Tropical Medicine, Tübingen, Germany, for technical assistance during the study period. The project received funding from a grant of the European Commission (TRANCHI; INCO-CT-2006-032436). polymorphisms. Hum Mol Genet 2003; 12: 2249-2254.

5. Nocentini G, Giunchi L, Ronchetti S, Krausz LT, Bartoli A, Moraca R, et al. A new member of the tumor necrosis factor/ nerve growth factor receptor family inhibits $T$ cell receptorinduced apoptosis. Proc Natl Acad Sci U S A 1997; 94: 6216-6221.

6. Kim JY, Moon SM, Ryu HJ, Kim JJ, Kim HT, Park C, et al. Identification of regulatory polymorphisms in the TNF-TNF receptor superfamily. Immunogenetics 2005; 57: 297-303. 